\title{
Desarrollo del sector financiero y su relación con el crecimiento de las micro y pequeñas empresas en Colombia (1995-2005) ${ }^{1}$
}

\section{Development of the financial sector and its relation with the growth of micro and small enterprises in Colombia (1995-2005)}

\author{
Marleny Cardona-Acevedo \\ Carlos Andrés Cano-Gamboa*
}

\begin{abstract}
The objective of this article is to verify the relation between some financial variables and industrial production in micro and small enterprises (Miypes) in Colombia, during the 1995-2005 period. To do so, we propose a model of panel data in order to establish the relation between gross production (dependent variable) and the following variables: number of enterprises, employed personnel, credit expenditure, active interest rate, as well as gross and nonperforming loans by enterprise size (micro and small). We found that the financial variable with the most positive effect on Miypes gross production is the variable of disbursements of Bancóldex, proxy variable of the government policies to foster entrepreneurial foment.
\end{abstract}

Keywords: financing policies, Colombian productive structure, Miypes, financial sector.

\section{Resumen}

El objetivo de este artículo es comprobar la relación entre algunas variables financieras y la producción industrial en las micro y pequeñas empresas (Miypes) en Colombia, durante el periodo 1995-2005. Para ello se propone un modelo datos de panel para establecer la relación entre la producción bruta (variable dependiente) y las variables número de establecimientos, personal ocupado, desembolso de créditos, tasa de interés activa y cartera vencida y bruta por tamaño de empresa (micro y pequeña). Se encontró que la variable financiera que tiene un efecto positivo en la producción bruta de las Miypes es la variable desembolsos de Bancóldex, variable proxy de las políticas del gobierno para incentivar el fomento empresarial.

Palabras clave: políticas de financiamiento, estructura productiva colombiana, Miypes, sector financiero.

\footnotetext{
${ }^{1}$ Este artículo es parte del trabajo "El mundo financiero en las micro y pequeñas empresas de Colombia (1995-2005)", realizado por el Grupo de Estudios Sectoriales y Territoriales (ESYT) de la Universidad EAFIT (Medellín, Colombia).

*Universidad EAFit, Colombia. Correos-e: marca@eafit.edu.co, ccanogam@eafit.edu.co.
} 


\section{Introducción}

El objetivo de este artículo es analizar la incidencia de las variables asociadas al sistema financiero en Colombia sobre la dinámica de la producción bruta de las Miypes. En general, la teoría económica analiza la relación entre el sector financiero y el crecimiento económico, pero poco se ha estudiado la relación del sector financiero, y específicamente la producción de las unidades productivas por tamaño de empresas. Este aspecto se destaca en este trabajo, intentando establecer la relación entre la producción industrial de las micro y pequeñas empresas y las variables financieras (profundización financiera, cartera vencida y cartera bruta, tasas de interés activas y evolución de los microcréditos).

Para el caso de Colombia se encontraron estudios similares, como el de Tenjo y García (1995). Este trabajo analizó la relación empírica entre un grupo de indicadores de desarrollo del sistema de intermediación financiera y el crecimiento económico. Desde esta perspectiva teórica relativamente estrecha, los resultados de los ejercicios econométricos apoyan la hipótesis de que existe una conexión significativa entre los aspectos financieros y los aspectos reales de la economía.

En efecto puede afirmarse, de acuerdo con los resultados de Tenjo y García (1995), que un mayor desarrollo de los servicios que el sistema bancario presta al sector privado, incluyendo los servicios tradicionales de intermediación y los relacionados con el manejo del riesgo y la información, propicia mayores tasas de crecimiento económico. Algo similar puede esperarse de una mayor capacidad del sistema para generar crédito y favorecer una mayor redistribución de éste al sector privado.

Los autores, por medio de mínimos cuadrados ordinarios, estimaron la tasa de crecimiento como un indicador de la profundización financiera, de la participación de los bancos domésticos en el total de activos del sistema y de la participación del crédito al sector privado en el total del crédito doméstico y el producto interno bruto (PIB). Además, con la información disponible para Colombia, se escogieron tres indicadores de crecimiento económico y se evaluó su relación con indicadores de desarrollo financiero.

Los tres indicadores de crecimiento fueron la productividad industrial, la formación bruta de capital fijo y el stock de capital. Estas variables no mostraron relaciones estadísticamente significativas con los cuatro indicadores financieros, es decir, la productividad industrial, la formación 
bruta de capital fijo y el stock de capital no parecen verse afectados por el desarrollo financiero. ${ }^{2}$

Este trabajo presenta avances frente al de Tenjo y García (1995). En primer lugar la metodología datos de panel permite mayor flexibilidad para modelar las diferencias de comportamiento entre las observaciones. Esto es importante para nuestro caso por las diferencias estructurales que presentan las variables. En segundo lugar, se tienen en cuenta variables adicionales, como la profundización financiera, los desembolsos de créditos de Bancóldex, la calidad de la cartera, la evolución de los microcréditos y la tasa de interés promedio activa para explicar el cambio en la producción. En tercer lugar, en este trabajo se hace un esfuerzo por presentar la relación entre variables financieras y la producción de las unidades micro y pequeñas empresas.

Este planteamiento es novedoso, en el sentido de que la mayoría de los trabajos aborda la causalidad existente entre la profundización del sistema financiero y el crecimiento económico, ${ }^{3}$ o abordan la temática desde el pIB total, y no se especifica la dinámica de acuerdo con el tamaño de la empresa. Este factor constituye un avance empírico, ya que las Miypes requieren un análisis puntual y específico.

Otro trabajo importante en Colombia es el de Carvajal y Zuleta (1997). Los autores evaluaron la hipótesis teórica de que el mejor desarrollo del sistema financiero estimula el crecimiento económico en el largo plazo. Concluyen que la apertura en la cuenta de capitales permite a las economías acercarse con mayor velocidad a su estado estacionario. Se encontró evidencia estadística para la hipótesis según la cual una mayor eficiencia del sistema financiero (relación entre el total de activos del sistema y los gastos administrativos y laborales) aumenta la formación bruta de capital fijo con un rezago de un año.

La evidencia empírica no es tan clara. Por un lado se encuentra una relación directa y estadísticamente significativa entre el desarrollo del sector financiero y el crecimiento económico. Por ejemplo, Greenwood y Jovanovic (1990) buscaron una aproximación a la determinación bilateral del desarrollo financiero y el crecimiento económico, dejando de lado la disyuntiva de quién fue primero. Su trabajo les permitió concluir

\footnotetext{
${ }^{2}$ De acuerdo con la evidencia estadística, los autores afirman que para el caso colombiano, el desarrollo financiero apoya el comportamiento de la producción. Si bien buscamos identificar los canales de transmisión, la falta de información y la escasez de series para indicadores como productividad industrial, formación bruta de capital fijo y stock de capital, la relación de éstas con los indicadores financieros no son estadísticamente significativas y no se pudo encontrar un mecanismo que satisfaga la relación entre el desarrollo financiero y el crecimiento económico.

${ }^{3}$ Robinson (1952), Patrick (1966), Lucas (1988), Chandavarkar (1992), McKinnon (1973), Levine $(1997,2004)$ y Destinobles (2007), entre otros, estudian la relación entre el sector financiero y el sector real de la economía, pero lo abordan a partir del piB total, y no se especifica la dinámica de acuerdo con el tamańo de la empresa.
} 
que el crecimiento económico es un promotor de los intermediarios financieros, quienes están encargados de asignar el capital, el cual tiene como consecuencia la aceleración del crecimiento.

López (2003) y Dabós (2008) también encontraron relación directa. Por un lado, López (2003) investigó la relación entre la intermediación crediticia llevada a cabo por las instituciones bancarias y la actividad económica en Venezuela, utilizando información trimestral para el periodo 1983-2002. Se encontró una relación positiva entre la tasa de variación del crédito y la tasa de variación del producto no petrolero. Aun cuando se mantiene positivo para todo el periodo objeto de estudio, este efecto se hace menor a partir de 1989, año en el cual se inició un conjunto de reformas en el sector financiero y en otros sectores de la economía. Un elemento que podría explicar la disminución de este efecto sería el aumento de la importancia de los préstamos de corto plazo en la cartera de crédito.

Por otro lado, Dabós (2008) sugiere, de acuerdo con la evidencia teórica y empírica para el caso de Argentina, que el desarrollo de los mercados de capitales y del sistema financiero contribuye significativamente al crecimiento económico de largo plazo. El planteamiento es que el desarrollo del mercado de capitales, como la regulación y la normatividad, contribuirá a generar un mayor crecimiento de largo plazo. Si se aplican políticas que favorezcan la regulación y el contexto macroeconómico es positivo, se logrará el desarrollo financiero, superando limitaciones de corto plazo.

Guryay et al. (2007) encontraron evidencia de causalidad entre el crecimiento económico y el desarrollo de los intermediarios financieros en Chipre. Los autores examinaron la relación entre el desarrollo financiero y el crecimiento económico por medio de un modelo ordinary least square estimation method (ols). Por otra parte, por medio de test de cointegración no encontraron evidencia de la causalidad, lo que permitió establecer una relación positiva entre el desarrollo de los intermediarios financieros y el crecimiento económico de largo plazo.

También se destacan los trabajos de Goldsmith (1969), King y Levine (1993), Levine y Zervos (1998), Levine et al. (2000) y Beck y Levine (2004). Estos autores encuentran una correlación positiva entre el desarrollo financiero, desde distintas variables como la productividad, los canales de crédito y el nivel de actividad económica.

Por su parte, Loayza y Ranciere (2002), mediante un panel de datos, no encontraron una relación significativa entre el desarrollo financiero, medido por pasivos líquidos y M2 (ambos como porcentaje del PIB), con el crecimiento del stock de capital. Por otro lado, Ramírez y Aquino (2004) evaluaron empíricamente la relación entre desarrollo financiero, medido como el crédito privado doméstico como porcentaje del PIB, con el cre- 
cimiento del stock de capital físico, utilizando el método generalizado de momentos (GMM) en un contexto de datos de panel dinámico para 16 países de América Latina, y para el periodo 1970-2000. Los resultados sugieren que hay un efecto positivo del crédito privado doméstico como porcentaje del PIB sobre el crecimiento del stock de capital, mientras que en periodos de crisis financiera dicha relación es negativa.

En Colombia se ha trabajado para apoyar el mejoramiento productivo desde el sistema financiero, aumentando el desembolso de créditos, la cartera y los recursos a las micro y pequeñas empresas. Sin embargo, es importante continuar el análisis sobre la relación de los recursos en el crecimiento de la economía del país, por tamaño de empresa. En este trabajo se parte de la base de que el sistema financiero tiene efectos en el sector real al transferir recursos desde donde hay superávit a sectores que los necesitan, además de la necesidad de generar políticas financieras dirigidas a la industria que generen las condiciones para el desarrollo.

El esquema del trabajo es el siguiente. La primera parte es la introducción. La segunda sección presenta el marco teórico. La tercera analiza el comportamiento de la estructura productiva colombiana, por tamaño de empresa. En la cuarta parte se analizan las políticas de fomento financiero a las Miypes en Colombia y la evolución del sistema financiero en el país. La quinta parte presenta el modelo, su formulación y resultados. Finalmente se presentan las conclusiones.

\section{Marco teórico}

La relación entre el crecimiento económico y el sistema financiero presenta dos enfoques teóricos. En el primero se parte de la hipótesis de que no existe causalidad entre el desarrollo del sector financiero y el crecimiento económico. Robinson (1952), Patrick (1966), Lucas (1988) y Chandavarkar (1992), entre otros, han planteado una relación inversa donde el crecimiento económico es el que afecta, por medio de factores reales, la eficiencia en los mercados financieros.

Robinson (1952) destaca el papel significativo que juega el espiritu anímico empresarial, que es el determinante del proceso de acumulación y crecimiento económico. Según este autor, hay causas distintas a las financieras que pueden propiciar el crecimiento en la economía: 1) las condiciones técnicas, la investigación y la mejora en la educación; 2) las condiciones competitivas en la economía; 3) el stock de capital inicial y las expectativas que se forman de la experiencia pasada; 4) las políticas de inversión que generan empleo, y 5) financiar la inversión.

Patrick (1966) hizo referencia a un proceso similar con el nombre de demand-following; en el cual el desarrollo del sistema financiero seguiría 
a la demanda de la economía real, es decir, una vez iniciado el proceso de crecimiento de las firmas, los empresarios incrementan sus necesidades de recursos (inversión y ahorro), vinculando por esta vía la intermediación del sistema financiero. Así, el desarrollo financiero es causado por el crecimiento económico y la demanda.

En el otro extremo del debate se ubican Schumpeter (1911), McKinnon (1973), Levine $(1997,2004)$ y Destinobles (2007), para quienes las diferencias en los niveles de desarrollo y crecimiento se pueden explicar con base en las condiciones financieras de las economías, por lo que se han centrado en el estudio de los factores que afectan directamente al sistema financiero y que causan un efecto indirecto en el desempeño económico de los países. Este enfoque plantea una relación directa entre el sistema financiero y el crecimiento económico, en la que el crecimiento es generado por la sofisticación y eficiencia en el sistema financiero. La principal hipótesis es que un sistema financiero más desarrollado afecta las decisiones de inversión y ahorro, mejorando la asignación de recursos en la economía y, con ello, impulsando el crecimiento económico.

Se distinguen cinco funciones que presta el sistema financiero a las economías, mediante las cuales se reducen los costos de transacción, de obtención de información y de hacer cumplir los contratos: 1) producción de información ex ante acerca de las posibles inversiones y asignaciones de capital; 2) monitoreo de las inversiones realizadas y de los gobiernos corporativos; 3) comercialización, diversificación y administración de riesgos; 4) movilización de ahorros, y 5) provisión de medios de pagos para facilitar el intercambio de bienes y servicios.

Los autores mencionados han demostrado la relación positiva que existe entre el desarrollo financiero de una economía y su crecimiento económico, donde se puede concluir, como menciona Miller (1998), que la proposición de que los mercados financieros contribuyen al crecimiento económico es demasiado obvia como para una discusión seria. La evidencia convincente de que el sistema financiero influye en el crecimiento de largo plazo, pone de manifiesto una necesidad imperiosa de investigar en los terrenos político, legal, regulatorio y en las determinantes de políticas del desarrollo financiero (Levine, 2004).

\section{Comportamiento de la estructura productiva Miypes colombiana}

Según la Ley 905 del año 2004, se entiende por micro (incluidas las empresas familiares) y pequeñas empresas, toda unidad de explotación económica, realizada por personas naturales o jurídicas, en actividades empresariales, agropecuarias, industriales, comerciales o de servicios, 


\section{Cuadro 1 \\ Definición de empresas por tamańo en Colombia* (según la Ley 905)}

\begin{tabular}{|c|c|c|c|}
\hline Tipo de empresa & $\begin{array}{l}\text { Porpersonal } \\
\text { ocupado }\end{array}$ & $\begin{array}{c}\text { Por activos totales } \\
(S M L)\end{array}$ & $\begin{array}{l}\text { Monto de activos } \\
\text { (dólares) }\end{array}$ \\
\hline Microempresa & Menos de 10 & Menos de 501 & Menos de 81,500 \\
\hline Pequeña & Entre 11 y 50 & Entre 501 y 5,001 & Menos de 810,000 \\
\hline Mediana & Entre 51 y 200 & Entre 5,001 y 30,000 & $\begin{array}{l}\text { Menos de } \\
4 ’ 860,000\end{array}$ \\
\hline Gran empresa & Más de 200 & Más de 30,000 & Más de 4’860,000 \\
\hline
\end{tabular}

Fuente: Elaboración propia a partir de la Ley 905.

* Para aquellas Miypes que presenten combinaciones de parámetros de planta de personal y activos totales diferentes a los indicados, el factor determinante para dicho efecto será el de activos totales.

rurales o urbanos, que respondan a dos de los siguientes parámetros (cuadro 1$){ }^{4}$

- Microempresa: a) planta de personal no superior a 10 trabajadores, o b) activos totales excluida la vivienda por valor inferior a 500 salarios mínimos mensuales legales vigentes, o 179 millones de pesos.

- Pequeńa empresa: a) planta de personal entre 11 y 50 trabajadores, o b) activos totales por valor entre 501 y menos de 5,000 salarios mínimos mensuales legales vigentes, o entre 179 y 1,790 millones de pesos colombianos.

De acuerdo con la información de la Encuesta Anual Manufacturera (ЕAM), 91.2\% de los establecimientos manufactureros son considerados pequeñas y medianas empresas (17.2 son microempresas, $49.8 \%$ son pequeñas y $24.2 \%$ son medianas), los cuales emplean $45.9 \%$ de los 587,630 trabajadores industriales registrados (de los cuales $1.4 \%$ labora en la microempresa, $14.8 \%$ son trabajadores de las pequeñas empresas y $29.7 \%$ se emplean en las medianas).

Las Miypes representan 67\% de los establecimientos industriales de Colombia, en el año 2005 generaron $10.7 \%$ de la producción bruta industrial y ocuparon $16.2 \%$ del personal de la industria manufacturera. Con respecto al valor agregado, las Miypes representaron 8.3\% del valor agregado del sector manufacturero. Adicionalmente, generaron $7.1 \%$ del total de activos industriales y $12.5 \%$ del consumo intermedio industrial (cuadro 2).

${ }^{4}$ Esta definición de la ley colombiana no tiene en cuenta la actividad que desempeñe la empresa. Lo ideal es que el tamaño de las compañías sea relativo a su propio sector. 

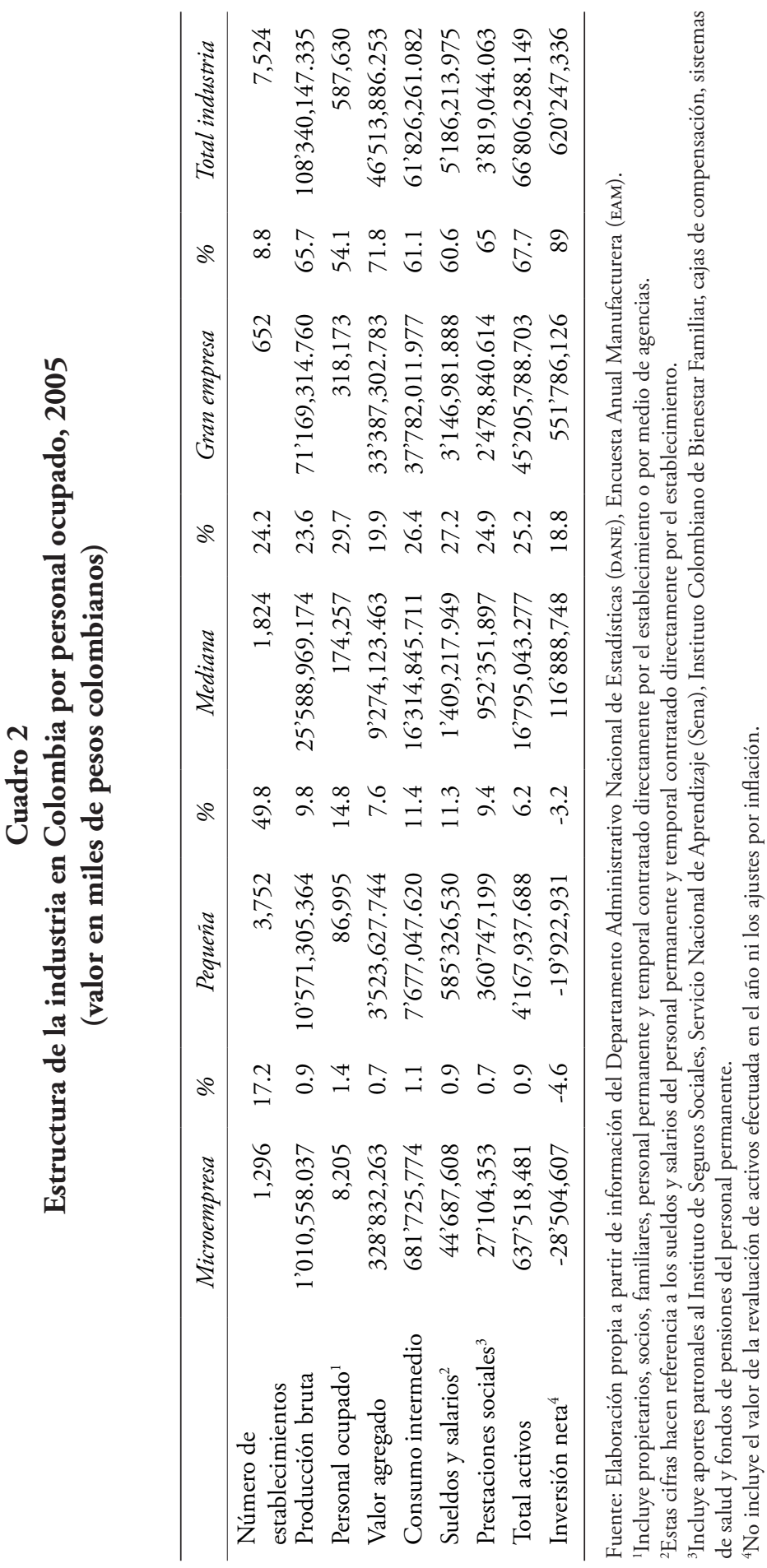


\section{Cuadro 3}

\section{Establecimientos en la industria colombiana por tamańo, 1990-2005 (porcentaje de participación)}

\begin{tabular}{lccccc}
\hline & Microempresas & Pequeñas & Medianas & Grandes & Total \\
\hline 1990 & 11 & 55 & 21 & 13 & 100 \\
1991 & 13 & 58 & 22 & 7 & 100 \\
1992 & 11 & 60 & 22 & 7 & 100 \\
1993 & 11 & 58 & 23 & 8 & 100 \\
1994 & 13 & 55 & 24 & 8 & 100 \\
1995 & 14 & 55 & 23 & 8 & 100 \\
Promedio 1990-1995 & 12 & 57 & 23 & 9 & 100 \\
1996 & 18 & 52 & 23 & 7 & 100 \\
1997 & 20 & 52 & 22 & 6 & 100 \\
1998 & 20 & 52 & 21 & 7 & 100 \\
1999 & 25 & 49 & 20 & 6 & 100 \\
2000 & 19 & 51 & 22 & 8 & 100 \\
Promedio 1996-2000 & 19 & 52 & 22 & 7 & 100 \\
2001 & 18 & 50 & 23 & 9 & 100 \\
2002 & 18 & 49 & 24 & 9 & 100 \\
2003 & 19 & 30 & 42 & 9 & 100 \\
2004 & 19 & 42 & 30 & 9 & 100 \\
2005 & 20.1 & 47.8 & 23.1 & 9 & 100 \\
Promedio 2001-2005 & 19 & 45 & 28 & 9 & 100 \\
\hline
\end{tabular}

Fuente: Elaboración propia a partir de información del DANE. Encuesta Anual Manufacturera.

Según datos de la EAM, la pequeña empresa en Colombia representaba $51.5 \%$ de los establecimientos industriales en el periodo 1990-2005, las medianas $24.1 \%$, las grandes empresas $8 \%$ en promedio, y las microempresas industriales $16.4 \%$ (cuadro 3). Las pequeñas y medianas empresas del sector manufacturero presentaron una etapa de crecimiento en el periodo postapertura, pasaron de 76\% en el año 1990 a $81 \%$ en 1993 . A partir de este año se presenta un menor nivel de representatividad, que se desplaza a la microindustria, segmento de mayor crecimiento en el país. Por su parte, la gran industria permanece en niveles constantes, alcanzando el nivel más alto en 1990 (13\%), después pierde representatividad, especialmente a partir de 1999.

La producción industrial en Colombia presenta un proceso de reconfiguración, en donde las Pymes ganan y pierden participación, sobre todo las medianas, ${ }^{5}$ y donde las grandes empresas pierden representatividad en el periodo 1996-2000. Por su parte, las micro y pequeñas empresas se mantienen en niveles similares de participación durante el periodo de

\footnotetext{
${ }^{5}$ En el cuadro 4 se muestra que la participación de la mediana empresa bajó a 20\% (1993) y subió a 31\% (2000), y la pequeña empresa movió su participación entre 9 (1993) y 22\% (2003).
} 


\section{Cuadro 4 \\ Producción industrial en Colombia según escala de personal ocupado (porcentajes)}

\begin{tabular}{lccccc}
\hline \multicolumn{1}{c}{ Año } & Microempresa & Pequeña & Mediana & Grande & Total \\
\hline 1991 & 2 & 11 & 25 & 62 & 100 \\
1992 & 2 & 13 & 26 & 59 & 100 \\
1993 & 1 & 9 & 20 & 70 & 100 \\
1994 & 2 & 13 & 25 & 60 & 100 \\
1995 & 1 & 12 & 26 & 61 & 100 \\
Promedio 1991-1995 & 2 & 11 & 24 & 63 & 100 \\
1996 & 2 & 12 & 27 & 59 & 100 \\
1997 & 2 & 12 & 27 & 59 & 100 \\
1998 & 3 & 13 & 28 & 56 & 100 \\
1999 & 3 & 14 & 28 & 55 & 100 \\
2000 & 3 & 15 & 31 & 51 & 100 \\
Promedio 1996-2000 & 3 & 13 & 28 & 56 & 100 \\
2001 & 1 & 9 & 25 & 65 & 100 \\
2002 & 1 & 9 & 22 & 68 & 100 \\
2003 & 2 & 22 & 21 & 65 & 100 \\
2004 & 2 & 18 & 23 & 57 & 100 \\
2005 & 1.7 & 14 & 26.8 & 57.2 & 100 \\
Promedio 2001-2005 & 1.4 & 14.5 & 23 & 63 & 100 \\
\hline
\end{tabular}

Fuente: Elaboración propia a partir de información del DANE. Encuesta Anual Manufacturera.

análisis (cuadro 4). En particular la dinámica de la gran empresa se da en el periodo 2001 y 2005, logrando mayor grado de participación en cuanto a la producción industrial.

El total de la pequeńa y mediana empresa representa $37.1 \%$ de la producción bruta (en promedio), alcanzando el nivel más alto en los años 1999 y 2000. La microempresa se mantiene en un rango de 1 y $3 \%$ (en promedio) en el periodo de análisis. La pequeña empresa (en promedio) llega a $12.6 \%$ de la producción bruta manufacturera.

En Colombia, el valor agregado se concentra en las grandes empresas (en promedio en el periodo 2001-2005 representó 63\%), pero ha perdido participación en el total del sector manufacturero. La mediana empresa ha incrementado el nivel de participación del valor agregado en el total de la industria manufacturera, pasó de $23 \%$ en 1990 a 30\% en el año 2005. Las pequeñas empresas representaban $9 \%$ en 2005 , cifra similar a la que tenían en 1990 (cuadro 5). Por otra parte, la generación de empleo en la industria manufacturera en las grandes empresas en los últimos 15 ańos tuvo una participación promedio de $48 \%$, con un aumento significativo en los últimos cinco años. Durante las últimas dos décadas las Pymes han generado cerca de 52\% del empleo manufacturero (cuadro 6). 
Cuadro 5

Valor agregado industrial de Colombia, 1990-2005 (porcentajes)

\begin{tabular}{lcccc}
\hline & Pequeñas & Medianas & Grandes & Total \\
\hline 1990 & 9 & 23 & 68 & 100 \\
1991 & 9 & 24 & 67 & 100 \\
1992 & 10 & 26 & 64 & 100 \\
1993 & 11 & 25 & 64 & 100 \\
1994 & 12 & 25 & 63 & 100 \\
1995 & 10 & 24 & 66 & 100 \\
Promedio 1990-1995 & 10 & 25 & 65 & 100 \\
1996 & 11 & 25 & 64 & 100 \\
1997 & 11 & 25 & 64 & 100 \\
1998 & 11 & 26 & 63 & 100 \\
1999 & 11 & 26 & 63 & 100 \\
2000 & 8 & 24 & 68 & 100 \\
Promedio 1996-2000 & 10 & 25 & 64 & 100 \\
2001 & 8 & 26 & 66 & 100 \\
2002 & 8 & 27 & 65 & 100 \\
2003 & 7 & 31 & 62 & 100 \\
2004 & 7 & 32 & 61 & 100 \\
2005 & 9 & 30 & 61 & 100 \\
Promedio 2001-2005 & 8 & 29 & 63 & 100 \\
\hline
\end{tabular}

Fuente: Elaboración propia a partir de información del DANE. Encuesta Anual Manufacturera.

\section{Cuadro 6}

Personal ocupado en la industria en Colombia, 1990-2005 (porcentajes)

\begin{tabular}{lccccc}
\hline & Microempresas & Pequeñas & Medianas & Grandes & Total \\
\hline 1990 & 1 & 21 & 30 & 48 & 100 \\
1991 & 1 & 20 & 31 & 48 & 100 \\
1992 & 2 & 20 & 30 & 39 & 100 \\
1993 & 2 & 19 & 30 & 49 & 100 \\
1994 & 2 & 18 & 31 & 49 & 100 \\
1995 & 3 & 18 & 31 & 48 & 100 \\
Promedio 1991-1995 & 2 & 19 & 31 & 47 & 100 \\
1996 & 4 & 18 & 31 & 47 & 100 \\
1997 & 4 & 19 & 31 & 46 & 100 \\
1998 & 4 & 19 & 31 & 46 & 100 \\
1999 & 6 & 19 & 32 & 43 & 100 \\
2000 & 2 & 16 & 29 & 53 & 100 \\
Promedio 1996-2000 & 4 & 18 & 31 & 47 & 100 \\
2001 & 2 & 15 & 29 & 54 & 100 \\
2002 & 1 & 15 & 30 & 54 & 100 \\
2003 & 1 & 14 & 32 & 53 & 100 \\
2004 & 1 & 16 & 33 & 50 & 100 \\
2005 & 1 & 15 & 30 & 54 & 100 \\
Promedio 2001-2005 & 1 & 15 & 31 & 53 & 100 \\
\hline
\end{tabular}

Fuente: Elaboración propia a partir de información del DANE. Encuesta Anual Manufacturera. 
En un balance respecto al empleo, se observa que esta variable ha presentado oscilaciones acentuadas en todos los tamaños de empresa en Colombia. Promediando el periodo 1990-1995, se aprecia que la pequeña empresa perdió participación en la generación de empleo con respecto al periodo preapertura económica, que comenzó en 1990 (pasó de 21 a 19\%, según datos del Departamento Nacional de Planeación, DNP), el cual absorbió en parte la mediana empresa. ${ }^{6}$

A partir de 1992, el empleo industrial presentó un cambio en su dinámica, las Pymes ganaron participación con $59 \%$ y las grandes empresas perdieron con 39\%, debido a los cambios en el modelo económico, ya que se permitió una liberalización comercial que generó alta competencia externa; por ende, se buscó mayor racionalización de costos en las empresas, con disminuciones en el empleo en términos absolutos. Este comportamiento continuó hasta 1995, con excepción del caso de la pequeña empresa, la cual presentó un crecimiento positivo de creación de empleo.

\subsection{Comportamiento de algunas variables financieras en Colombia}

En este trabajo, al tener como sujeto de estudio a las pequeñas y medianas empresas, se destaca que es importante observar la dinámica del crédito dentro del sistema financiero en Colombia en los últimos años. La tasa de interés de captación o de los certificados a término fijo (DTF) ha tenido tres momentos claramente diferenciables en este país.

El primero de 1986 a 1991, donde se presentó un comportamiento relativamente estable con una tasa promedio de $34 \%$. Un segundo momento se dio después de 1991, cuando se formalizan los cambios normativos y la autonomía e independencia del Banco de la República y su objetivo definido de control de nivel de precios. A partir de entonces comenzó el proceso de reducción de la inflación, así la DTF experimentó una disminución en promedio de $29 \%$ hasta 1998 . El tercer momento se presenta a finales de 1998, cuando la DTF comienza una caída constante (gráfica I). En este periodo, la DTF pasó de niveles de 37.5\% en noviembre de 1998 a $10 \%$ en un lapso de 14 meses. Actualmente la DTF está en el 3.96\% efectivo anual.

\footnotetext{
${ }^{6}$ Lo anterior se debe a que mientras en el periodo de preapertura la pequeńa empresa mostró mayor dinamismo en el empleo industrial (crecimiento anual promedio de 3\%), en el periodo de postapertura el empleo de la mediana empresa tuvo un mayor crecimiento $(2.4 \%$ promedio anual, frente a $2.1 \%$ de la gran empresa y $-0.9 \%$ de la pequeña).
} 


\section{Gráfica I}

\section{Tasa de interés de los certificados de depósito a 90 días (DTF) en Colombia, 1986-2006*}

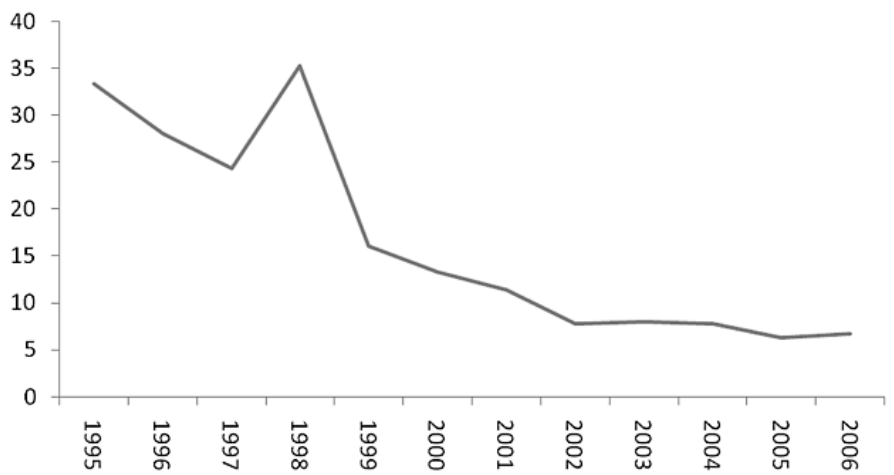

Fuente: Elaboración propia con información de la Superintendencia Financiera de Colombia.

* A junio de 1993 corresponde la encuesta diaria promedio de bancos y corporaciones, realizada por el Banco de la República. En adelante, corresponde a las tasas de captación de CDT a 90 días, promedio mensual ponderado, informada por bancos, corporaciones financieras, corporaciones de ahorro y vivienda y compañías de financiamiento comercial de todo el país a la Superintendencia Bancaria para el cálculo de la DTF.

\section{Gráfica II}

\section{Nivel de endeudamiento: micro y pequeñas empresas en Colombia,} 1989-2005

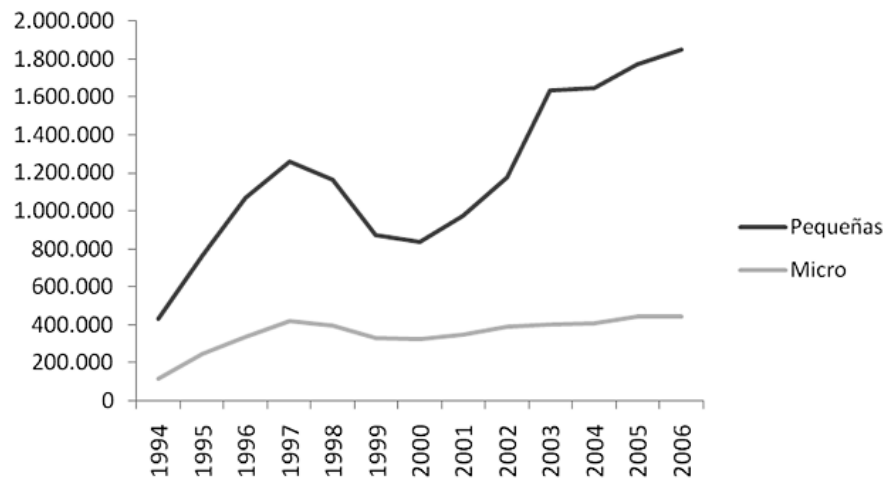

Fuente: Elaboración propia con información del Banco de la República. Valor en miles de millones de pesos en moneda constante. 
Después de las reformas, el endeudamiento de las micro y pequeñas empresas tuvo un incremento significativo entre 1994-1998 y una fuerte disminución debido a la crisis económica de 1999 en el país. En los últimos años, con la recuperación financiera y el periodo de auge a partir de 2002 se presenta una mejoría en el crédito adquirido por la industria micro y pequeña (gráfica II).

La relación del crecimiento en el sector financiero y el pIB es una forma de comenzar a indagar sobre una correlación que permita corroborar que el sistema financiero tiene efectos positivos en el crecimiento económico, como se plantea teóricamente. En Colombia, con el fin de enfrentar la crisis que comenzó en 1999, la banca central emprendió una serie de cambios en la forma institucional de la moneda.

Algunos de esos cambios, de acuerdo con Escovar y González (2004), fueron: 1) la implementación de la multibanca; 2) la liberación de las tasas de interés para que fuera el mercado quien asignara tanto las tasas de captación como de colocación; 3) se eliminaron controles cuantitativos al crédito; 4) se redujeron y simplificaron las inversiones forzosas y sustitutivas del encaje; 5) para disminuir los riesgos se exigió mayor capital y un nivel de solvencia mínimo; 6) se aumentó el nivel para la calificación de la cartera y se fortalecieron las reglas para la provisión de cartera vencida; 7) se prohibió que las entidades financieras estén cortas de moneda extranjera; 8) la reforma cambiaria convirtió al sistema financiero en el principal intermediario en el mercado de divisas, y 9) la liberalización de las tasas de interés.

La profundización financiera ${ }^{7}$ se relaciona con los planteamientos de Schumpeter (1911) cuando argumenta que el indicador del nivel de desarrollo financiero está estrechamente relacionado con el crecimiento. Existe mayor grado de profundización financiera cuando aumenta el volumen de las transacciones realizadas por los agentes económicos por medio de la banca. Para medir el grado de profundización financiera se construye la relación M3/PIB. La disminución de la relación demuestra una menor profundización financiera.

La gráfica III muestra que en Colombia se presentó una menor profundización financiera a partir de 1996 hasta 2005, año en el que se empieza a revertir esta tendencia. Sin duda, la profundización en Colombia es muy baja, lo que disminuye la capacidad que tiene el sector financiero para generar crecimiento económico. Se ha comprobado que un mayor acceso a estos servicios, tanto por parte de las medianas, pequeñas

\footnotetext{
${ }^{7}$ Ésta se mide como participación del agregado monetario $\mathrm{M}_{3}$ en el pIв. Si este indicador presenta aumento significa una mejor asignación de recursos a los sectores rentables. El nivel de desarrollo económico, el nivel de infraestructura financiera y los mecanismos de regulación son los factores que influyen en el grado de profundización de un sistema financiero.
} 


\section{Gráfica III \\ Profundización financiera en Colombia, 1995-2006}

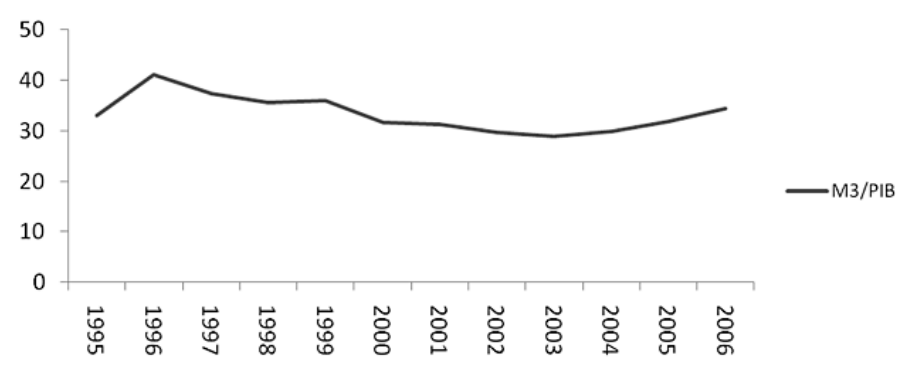

Fuente: Elaboración propia con información del Banco de la República.

y micro empresas, como de las familias, se relaciona positivamente con un mayor crecimiento económico.

King y Levine (1993) han demostrado la relación directa entre la profundización financiera y el crecimiento económico. Un sistema financiero eficiente aumenta la inversión porque canaliza recursos al sector real, disminuye costos de transacción y financieros, probablemente incentiva el ahorro en los hogares y diversifica los instrumentos de activos financieros para el acceso al crédito.

La cartera es una variable explicativa del riesgo. El comportamiento de la cartera en el sistema financiero ha crecido a partir de 1990 con los diferentes cambios estructurales presentados. Entre 1998 y 2003 tuvo una disminución como efecto de la crisis económica (gráfica IV). La calidad de la cartera se mide como la relación entre la cartera vencida y la cartera bruta. Si este indicador presenta aumento, significa que existe una calidad de la cartera deficiente, es decir, cartera vencida y con mayor riesgo de pérdida. En Colombia, la calidad de la cartera se vio afectada hasta el año 2001 como respuesta a los años de la crisis (gráfica v).

\section{Formulación del modelo}

En este estudio partimos de la hipótesis de que el sistema financiero influye en el crecimiento económico en el largo plazo. En el caso específico de la consolidación de las micro y pequeñas empresas, se considera a los servicios financieros como dinamizadores de las estructuras productivas. La relación de las variables explicativas con la producción bruta busca comprobar si la profundización del sistema financiero tiene efectos en el crecimiento de la micro y pequeña empresa en Colombia.

El análisis se basa en los signos y significancia de los coeficientes, a partir de los cuales se verifica la relación entre las variables que señalan 


\section{Gráfica IV \\ Comportamiento de la cartera neta en Colombia, 1990-2006}

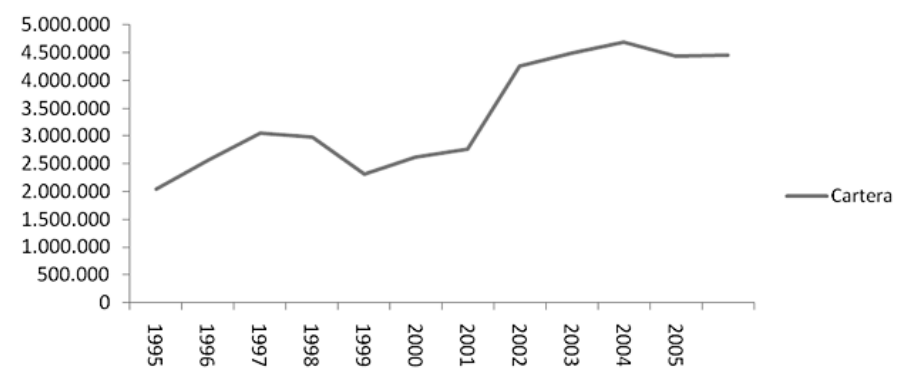

Fuente: Elaboración propia con información del Banco de la República.* Valor en miles de millones de pesos colombianos.

* A partir del 29 de junio de 2001 se presentan algunas modificaciones en las estadísticas de los agregados monetarios y crediticios, las cuales se efectuaron para incorporar transformaciones recientes del sistema financiero colombiano. En particular, dichas modificaciones se realizaron para dar un tratamiento especial a las entidades en liquidación, incluir los CDT emitidos por entidades financieras especiales, y los depósitos a la vista de las entidades de depósito no bancarias. De esta manera, en la nueva serie de medios de pago (M1) se excluyen las cuentas corrientes de las entidades en liquidación y se modifica la serie de efectivo. Por su parte, en el cómputo del agregado M3, se excluyen de los pasivos sujetos a encaje los depósitos de las entidades en liquidación, los cuales se presentan por separado dentro de dicho agregado y se identifican como depósitos restringidos. La revisión de M3 incluye también los CDT emitidos por entidades financieras especiales, los depósitos a la vista de las entidades de depósito no bancarias, así como una cooperativa que fue autorizada como ente depositario. De igual forma, las series de cartera e inversiones del sistema financiero, tratan por separado las operaciones de las entidades en liquidación. Dado que la información de las entidades en liquidación fue actualizada, los saldos de cartera e inversiones presentan algunos cambios. Las series históricas fueron corregidas para mantener las consistencias.

\section{Gráfica V \\ Calidad de las carteras vencida y bruta en Colombia, 1995-2006}

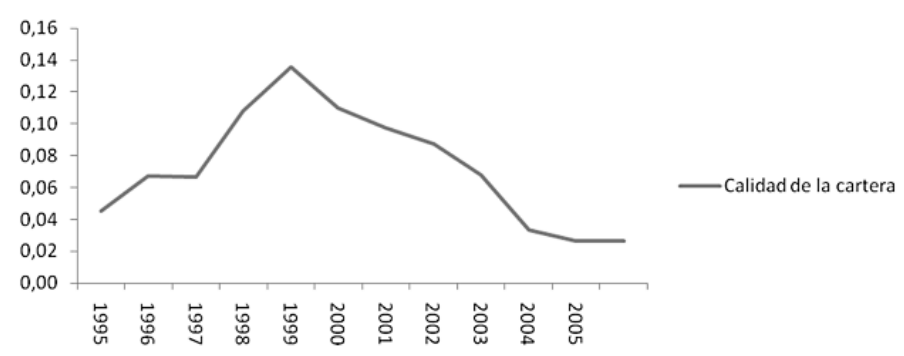

Fuente: Elaboración propia con información de la Superintendencia Financiera, 2007. 
los teóricos desde dos enfoques: 1) Schumpeter (1911), Goldsmith (1969), McKinnon (1973) y Levine (1997) establecieron una relación positiva entre el nivel de desarrollo del sector financiero y el crecimiento del sector real, en este caso de las micro y pequeńas empresas; y 2) la teoría austriaca que destaca la importancia de la tasa monetaria de interés y su influencia en las decisiones acerca del ahorro y la inversión.

En este estudio sobre el mundo financiero y su relación con el sector real, se hace un análisis econométrico que permite indagar cuál es la relación entre algunas variables financieras y la producción industrial en las Miypes de Colombia en el periodo 1995-2005.

El modelo es el siguiente:

$$
\Delta P B_{i t}=\alpha+\beta_{X_{i t}}+\gamma_{t}+\eta_{i}+v_{i t}
$$

Donde $i$ representa el tamaño de empresa: micro y pequeña; $t$ es el periodo; $\Delta P B_{i t}$ es la tasa de la variación de la producción bruta por tamaño de empresa (micro y pequeña). Muestra el efecto de las economías externas de urbanización, la industria en un área donde existe mano de obra diversificada que puede incrementar la productividad del sector. Esta información se obtiene de la Encuesta Anual Manufacturera del DANE. $X_{i t}$ representa el vector de variables explicativas.

- Número de establecimientos y personal ocupado: determinan la evolución de la dinámica interna del tipo de unidad productiva. Se esperaría un signo positivo, partiendo de que un incremento de la actividad productiva incidiría en la producción bruta. Esta información se obtiene de la Encuesta Anual Manufacturera.

- Desembolso de crédito: Créditos otorgados por Bancóldex a las micro y pequeñas empresas de Colombia. Se esperaría un signo positivo, ya que la canalización de recursos del sector financiero al sector real permitiría mayor liquidez y dinámica en la producción.

- Tasa de interés: variación de la tasa DTF. Se esperaría un signo negativo. Un incremento de la tasa de interés incide en el costo del uso del capital, por lo que desincentiva la producción.

- Cartera vencida: monto en millones de pesos de la cartera vencida de las micro y pequeñas empresas de los créditos otorgados por Bancóldex. Se esperaría signo negativo en el modelo. La calidad de la cartera incidiría en la capacidad de otorgar liquidez al sistema por parte del sistema financiero. 
$\gamma_{t}$ representa el efecto temporal y $\eta_{i}$ es el efecto individual no observable.

El modelo tiene como variable dependiente la producción bruta de las Miypes. Las variables explicativas representan las dos variables dominantes de los movimientos de la producción: capital y trabajo. Por el lado del capital tenemos los desembolsos de créditos, y por el del trabajo el personal ocupado. Además, se tienen presente las variables financieras DTF y la calidad de la cartera.

El periodo de análisis (1995-2005) se restringe a la información que suministran las instituciones encargadas del desarrollo y fortalecimiento de las Miypes en Colombia. Se tiene presente que este periodo abarca dos condiciones estructurales de la economía colombiana: la apertura comercial y la recesión de la economía en 1999-2000.

El modelo considera que los errores variantes a lo largo del tiempo no se correlacionan con valores presentes y pasados de ciertas variables condicionantes, de modo que éstas las predeterminan los errores variantes en el tiempo. Los resultados del modelo fueron validados mediante las pruebas de los errores estándar robustos para heterocedasticidad, la probabilidad de los coeficientes, el test de especificación y de selección entre efectos fijos o aleatorios de Hausman y Taylor ${ }^{8}$ (1981). Los modelos cumplen con los supuestos básicos que validan el uso de la técnica de datos de panel (por efectos fijos), por lo que las estimaciones son consistentes.?

La construcción de la base de datos que estableciera relaciones se conceptualizó y construyó con información obtenida de Bancóldex, el Banco de la República y el Departamento Administrativo Nacional de Estadísticas (DANE) con el criterio de los códigos de las agrupaciones industriales y escala de personal ocupado. En la estructuración de la base de datos para la modelación se encontraron algunas dificultades en la calidad y cantidad de los datos, limitando el tipo de método que se podría utilizar al realizar las predicciones que permitieran analizar la estructura regional de las Miypes y abarcar un periodo de análisis más largo.

\subsection{Resultados del modelo}

Los resultados indicarían la existencia de una relación negativa entre la DTF y la producción bruta de las micro y pequeñas empresas. Ésta es la relación esperada porque a mayor tasa de interés más se desincentivaría

\footnotetext{
${ }^{8}$ El test de especificación de Hausman se utiliza para verificar la ortogonalidad de los efectos aleatorios y los regresores (Greene, 2003).

${ }^{9}$ Se verifica la no existencia de autocorrelación en el término de error, lo que indica que su distribución es ruido blanco. Los resultados incluyen estimaciones en desviaciones ortogonales. Los errores estándar son robustos para heterocedasticidad.
} 
la generación de recursos encaminada a la producción industrial. Un incremento de $1 \%$ de la DTF generaría una disminución de la producción bruta de las pequeñas empresas de $0.59 \%$, mientras que la magnitud sería de $0.17 \%$ en las microempresas.

Con el número de establecimientos (NE), personal ocupado (po) y desembolso Bancóldex (DB) también se presenta la relación esperada: se muestra un efecto positivo con la variable dependiente. En las microempresas el efecto del incremento del número de establecimientos es mayor que en la pequeña empresa. Un incremento de $1 \%$ en el número de establecimientos generaría un efecto de crecimiento en la producción bruta de las pequeñas en $0.08 \%$, mientras que en las microempresas el efecto sería de $0.88 \%$. Con respecto al personal ocupado, un incremento de $1 \%$ generaría un aumento de $0.14 \%$ en la producción bruta de las microempresas, y de $0.42 \%$ de las pequeñas. Los desembolsos tienen una relación positiva con la producción, especialmente en las pequeñas empresas.

Aumento de desembolso de créditos para las micro y pequeñas empresas en Colombia. Cuando se incrementa en 1\% los desembolsos de créditos, se genera un efecto positivo de incremento en la producción de $0.47 \%$, mientras que en las microempresas este efecto sería de $0.24 \%$. El efecto de la cartera vencida sería muy similar en ambos tamaños de empresa. Tanto para las micro como para las pequeñas empresas se presentaría un incremento de la producción bruta, en las micro de $0.17 \%$ y en las pequeńas de $0.16 \%$, cuando se incrementa en $1 \%$ la cartera vencida. No se esperaba este signo ni su magnitud.

\section{Consideraciones finales}

De acuerdo con Goldsmith (1969), King y Levine (1993), Levine (1997, 2004) y con base en los resultados obtenidos en el modelo del presente trabajo, se podría afirmar que, para el caso de la economía colombiana, existe una relación estadísticamente significativa entre la actividad de intermediación crediticia, medida con la cartera de crédito de la banca, la tasa de interés y los desembolsos de créditos y la producción de las Miypes.

En la búsqueda de la interacción de las micro y pequeñas empresas con el sistema financiero en el periodo 1995-2005 se encontró que:

- La variable financiera que tiene un efecto positivo en la producción bruta de las micro y pequeñas empresas son los desembolsos de créditos por parte de Bancóldex (DB), con mayor incidencia en la producción bruta de las pequeñas empresas. 


\section{Resultados de las estimaciones del modelo}

\begin{tabular}{lcc}
\hline $\begin{array}{l}\text { Variable dependiente: producción bruta micro } \\
\text { y pequeñas empresas }\end{array}$ & Microempresas & Pequeñas empresas \\
\hline Variable & & \\
Tamaño & & \\
\hline Número de establecimientos (NE) & 0.887541 & 0.086891 \\
B & 1.658542 & 1.350585 \\
t & 0.0000 & 0.0347 \\
Prob & & \\
Personal ocupado (PO) & 0.146084 & 0.420082 \\
B & 0.616530 & 0.724434 \\
t & 0.0000 & 0.0012 \\
Prob & & \\
Tasa de interés (DTF) & -0.175563 & -0.590044 \\
B & -0.731139 & -3.427483 \\
t & 0.0000 & 0.0187 \\
Prob & & \\
Cartera vencida (CV) & & \\
B & 0.178674 & 0.160432 \\
t & 1.420425 & 1.86539 \\
Prob & 0.0000 & 0.0000 \\
Desembolsos Bancóldex (DB)) & & \\
B & 0.24317 & 0.47324 \\
t & 2.14006 & 3.15267 \\
Prob & 0.0000 & 0.0000 \\
Dw & & \\
R2 Ajustado & 2.129745 & 2.336035 \\
& 0.764798 & 0.837583 \\
\hline
\end{tabular}

- La cartera vencida presenta una relación directa, significativa y positiva con la producción, particularmente con mayor efecto en las microempresas.

- La variable financiera que tiene efectos negativos en la producción bruta de las micro y pequeñas empresas es la DTF, especialmente en las pequeñas empresas. La variación de la tasa de interés afectaría la producción de las Miypes, es decir, en Colombia existe una alta sensibilidad de las unidades productivas a la variación de la tasa de interés de sus créditos.

En este trabajo se considera una relación positiva entre el desarrollo financiero de una economía y el crecimiento económico, especialmente en las unidades productivas de menor tamaño, dadas sus condiciones de escasez de recursos de capital de trabajo. Así, los sistemas financieros pueden incidir en la tasa de ahorro, las decisiones de inversión, la innovación tecnológica y, por ende, en el crecimiento de largo plazo. 
La utilización amplia y profunda de servicios financieros conduce a una adecuada canalización de ahorros a actividades productivas, un eficiente sistema de pagos que facilita las transacciones entre agentes económicos y un adecuado monitoreo de los riesgos empresariales. Sin duda, la profundización financiera promueve el desarrollo de los microcréditos.

Como objeto de estudio se consideraron las micro y pequeńas empresas. El análisis de los resultados presentados muestran el rol importante del sistema financiero en el fortalecimiento de las micro y pequeñas empresas en Colombia, resaltando que los desembolsos tienen efectos positivos, y que el costo del uso del capital incide negativamente, en especial en las pequeñas empresas.

\section{Cuadro 7}

Instrumentos financieros de fomento Miypes en Colombia

\begin{tabular}{|c|c|c|}
\hline Instituciones & Servicios prestados & Ejecución \\
\hline Bancóldex & $\begin{array}{l}\text { Aparte de las líneas de redes- } \\
\text { cuento, Bancóldex desarro- } \\
\text { lla operaciones de factoraje } \\
\text { con microempresas y Pymes, } \\
\text { con el objeto de facilitarles } \\
\text { obtener liquidez inmediata } \\
\text { y mejorar su flujo de caja. En } \\
\text { la actualidad, el tipo de } \\
\text { factoraje que ofrece Bancól- } \\
\text { dex es en mayor parte para } \\
\text { el sector exportador y está } \\
\text { respaldado por compańías } \\
\text { aseguradoras. } \\
\text { Sin embargo, es necesario } \\
\text { desarrollar y masificar este } \\
\text { mercado para que las mi- } \\
\text { croempresas y las Pymes, } \\
\text { tanto exportadoras como no } \\
\text { exportadoras, puedan acce- } \\
\text { der efectivamente a este } \\
\text { instrumento de financia- } \\
\text { miento }\end{array}$ & $\begin{array}{l}\text { En el caso de Bancóldex, los } \\
\text { desembolsos a estos segmen- } \\
\text { tos empresariales aumentaron } \\
\text { en } 570 \% \text { entre } 2002 \text { y } 2006 \text {, } \\
\text { lo cual representó un incre- } \\
\text { mento de la participación de } \\
\text { esos sectores en el total de } \\
\text { desembolsos de } 8 \text { a } 59 \% \text { entre } \\
2002 \text { y } 2006\end{array}$ \\
\hline
\end{tabular}


continúa Cuadro 7 ...

\begin{tabular}{|c|c|c|}
\hline Instituciones & Servicios prestados & Ejecución \\
\hline Banco de las oportunidades & $\begin{array}{l}\text { Los resultados indican que } \\
\text { en los últimos años se logró } \\
\text { expandir y profundizar de } \\
\text { manera significativa la colo- } \\
\text { cación de crédito por parte } \\
\text { de bancos, cooperativas y } \\
\text { ONG }\end{array}$ & $\begin{array}{l}\text { Entre agosto de } 2006 \text { y agos- } \\
\text { to de } 2007 \text { se otorgaron } \\
\text { l'251,222 microcréditos por } \\
\text { valor de } 3.9 \text { billones de pesos } \\
\text { y se instituyeron } 147 \text { corres- } \\
\text { ponsales no bancarios y } 3,392 \\
\text { puntos de atención Citibank } \\
\text { a lo largo del territorio nacio- } \\
\text { nal. Además, se logró impul- } \\
\text { sar otros servicios financieros } \\
\text { para microempresarios, como } \\
\text { las cuentas de ahorro de bajo } \\
\text { monto }\end{array}$ \\
\hline $\begin{array}{l}\text { Fondo Nacional de } \\
\text { Garantías }\end{array}$ & $\begin{array}{l}\text { Para las líneas de crédito de } \\
\text { largo plazo, Bancóldex fijó } \\
\text { tasas de interés más bajas } \\
\text { frente a las demás líneas, con } \\
\text { el objeto de ofrecer un mar- } \\
\text { gen de intermediación que } \\
\text { incentive su colocación por } \\
\text { parte de los intermediarios } \\
\text { financieros }\end{array}$ & $\begin{array}{l}\text { El crédito movilizado con } \\
\text { garantía del FNG creció } \\
196 \% \text { y el número de mi- } \\
\text { croempresas y Pymes bene- } \\
\text { ficiadas pasó de } 50 \text { mil en } \\
2002 \text { a } 173 \text { mil en } 2006 \text {. De } \\
\text { igual manera, Bancóldex y el } \\
\text { FNG desarrollaron un esque- } \\
\text { ma de garantías compartidas } \\
\text { que permite garantizar hasta } \\
70 \% \text { de los créditos de largo } \\
\text { plazo }\end{array}$ \\
\hline Fomipyme & $\begin{array}{l}\text { El objetivo es cofinanciar } \\
\text { programas, proyectos y acti- } \\
\text { vidades para el desarrollo } \\
\text { tecnológico de las Mipymes } \\
\text { y la aplicación de instru- } \\
\text { mentos no financieros diri- } \\
\text { gidos a su fomento y promo- } \\
\text { ción }\end{array}$ & $\begin{array}{l}\text { El Fondo Colombiano de } \\
\text { Modernización y Desarrollo } \\
\text { Tecnológico de las Micro, } \\
\text { Pequeńas y Medianas Em- } \\
\text { presas (Fomipyme) cofinan- } \\
\text { ció } 320 \text { proyectos producti- } \\
\text { vos por un valor de } 58.5 \text { mil } \\
\text { millones de pesos, de los } \\
\text { cuales } 81 \% \text { se dirigió a aten- } \\
\text { der microempresas y } 19 \% \text { a } \\
\text { apoyar Pymes }\end{array}$ \\
\hline
\end{tabular}

Fuente: Elaboración propia con base en las instituciones reseñadas. 


\section{Gráfica VI}

\section{Desembolso total de crédito a las Miypes en Colombia, 1995-2007}

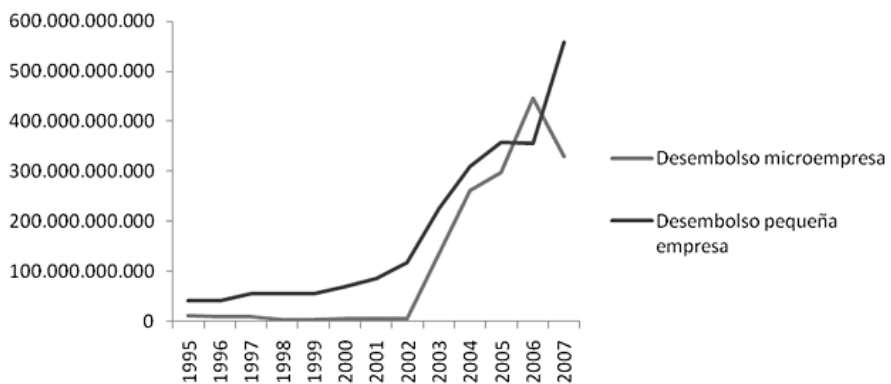

Fuente: Elaboración propia. Valor en miles de millones de pesos colombianos en moneda constante.

\section{Datos estilizados de las variables del modelo}

\section{Gráfica VII \\ DTF a 90 días}

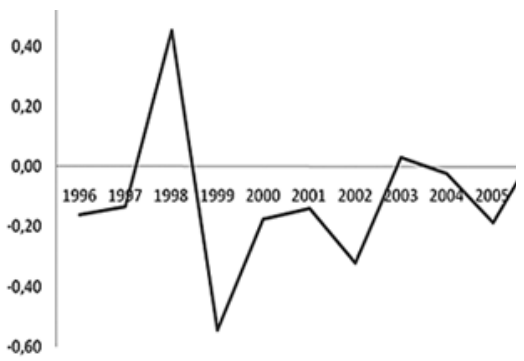

Fuente: Elaboración propia con información del Banco de la República.

Gráfica IX

Cartera vencida

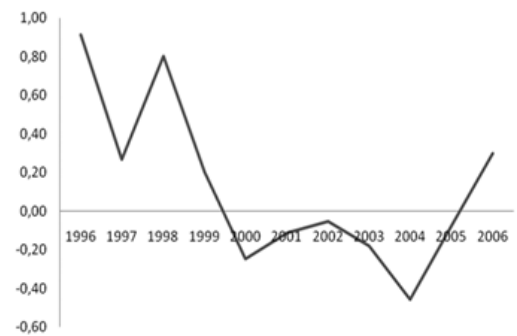

Fuente: Elaboración propia con información del Banco de la República.

\section{Gráfica VIII PIB financiero}

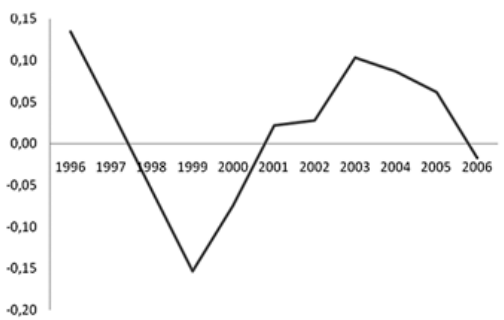

Fuente: Elaboración propia con información del Banco de la República.

\section{Gráfica x}

Profundización financiera

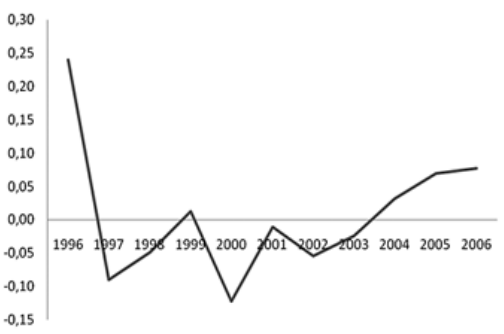

Fuente: Elaboración propia con información del Banco de la República. 
Gráfica XI

Personal ocupado en las microempresas

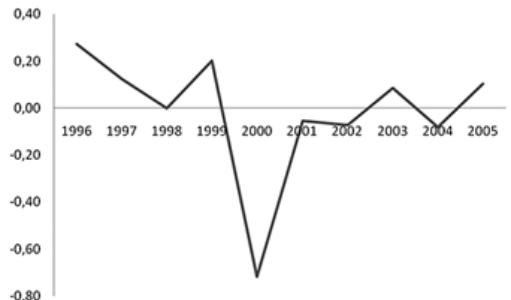

Fuente: Elaboración propia con información del Banco de la República.

Gráfica XIII

Número de establecimientos microempresas

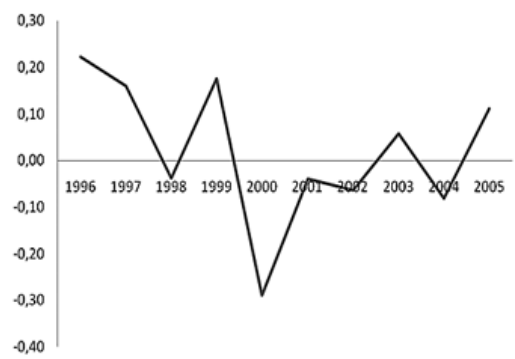

Gráfica XII

Personal ocupado en las pequeñas empresas

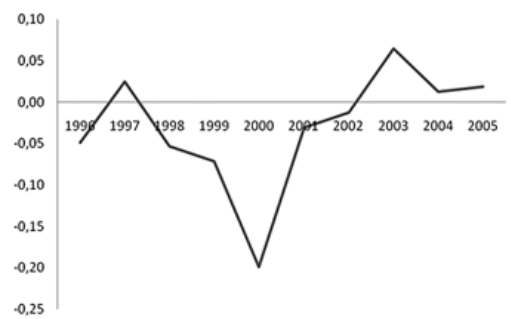

Fuente: Elaboración propia con información del Banco de la República.

Gráfica XIV

Número de establecimientos pequeńas empresas

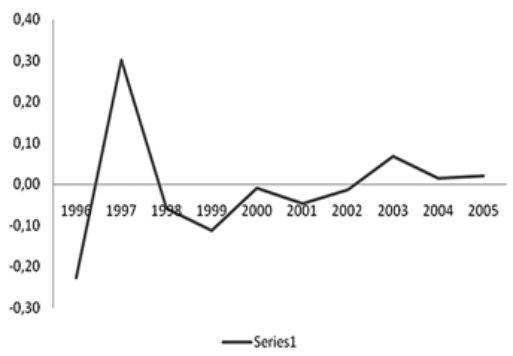

Fuente: Elaboración propia con información del Fuente: Elaboración propia con información del Banco de la República. Banco de la República.

\section{Gráfica XV}

\section{Producción bruta microempresas}

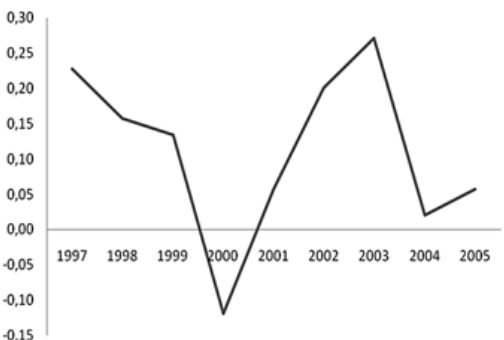

Gráfica XVI Producción bruta pequeñas empresas

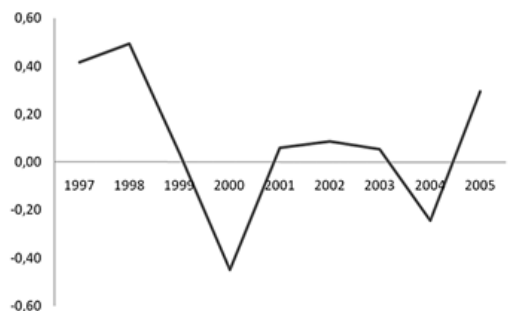

Fuente: Elaboración propia con información del Fuente: Elaboración propia con información del Banco de la República.

Banco de la República. 


\section{Bibliografía}

Beck, Thorsten y Ross Levine (2000), "New firm formation and industry growth-does having a market- or bank-based system matter?", Policy Research Working Paper Series 2383, World Bank, Washington.

Carvajal, Andrés y Hernando Zuleta (1997), "Desarrollo del sistema financiero y desarrollo económico", Borradores de Economía, 67, Bogotá, pp. 1-62.

Chandavarkar, Anand (1992), "Of finance and development: neglected and unsettled questions", World Development, 20 (1), Pergamon Press, Londres, pp. 133-142.

Dabós, Marcelo Pedro (2008), Mercado de capitales, sistema financiero y crecimiento económico, ADEBA, Buenos Aires.

Destinobles, Gerald (2007), Introducción a los modelos de crecimiento económico exógeno y endógeno, <www.eumed.net/libros/2007a/ 243/>, 21 de noviembre de 2008.

Escovar, Andrea y Gabriel Felipe González (2004), Una interpretación del sistema financiero colombiano desde la teoría de la regulación (19902000), Universidad EAFIT, Medellín.

Goldsmith, Raymond (1969), The quantitative of Financial structure and Development, <http://www.jstor.org/view/00220507/di975630 /97p0796f/0?frame=noframe\&userID=c80cb288@eafit.edu.co/ 01cc99332100501b7be37\&dpi=3\&config=jstor $>, 10$ de septiembre de 2008.

Greene, William H. (2003), Econometric Analysis, Prentice Hall, New Jersey.

Greenwood, Jeremy y Boyan Jovanovic (1990), "Financial Development, Growth, And The Distribution Of Income", The Centre for the Study of International Economic Relations, Working Papers 9002, University of Western Ontario, Ontario.

Guryay, Erdal, Okan Veli Şafakli y Behiye Tüzel (2007), "Financial Development and Economic Growth: Evidence from Northern 
Cyprus", International Research Journal of Finance and Economics, 8, EuroJournals Publishing, Inc., pp. 57-62.

Hausman, Jerry A. y William E. Taylor (1981), "Panel data and unobservable individual effects", Econometria, 49, Journal of the Econometria, E.U., pp. 1377-1398.

King, Robert G. y Ross Levine (1993), "Finance and Growth: Schumpeter Might Be Right", The Quarterly Journal of Economics, 108 (3), The мIт Press, Massachusetts, pp. 717-737.

Levine, Ross (1997), "Desarrollo financiero y crecimiento económico: puntos de vista y agenda", The Economic Journal, 35 (2), University of Virginia, Virginia, pp. 688-726.

Levine, Ross (2004), Finance and growth: theory and evidence, National Bureau of Economic Research, <http://www.nber.org/papers/ w10766>, actualización mayo de 2007, 2 de septiembre de 2008.

Levine, Ross, Norman Loayza y Thorsten Beck (2000), "Financial Intermediation and Growth: Causality and Causes", University of Chicago Press, Chicago, pp. 31-77.

Levine, Ross y Sara Zervos (1998), "Stock Markets, Banks and Economic Growth”, Policy Research Working Paper, 1690, The World Bank Policy Research Department, Finance and Private Sector Development Division.

Loayza, Norman y Romain Ranciere (2002), "Financial Development, Financial Fragility, and Growth", Banco Central de Chile, Documento de Trabajo, núm. 145.

Lucas, Robert (1988), "On the mechanics of Economic Development", Journal of Monetary Economics, 22, North Holland, Cambridge, pp. 3-42.

López, Antonio (2003), Intermediación crediticia y actividad económica en Venezuela, Banco Central de Venezuela, Caracas.

Mckinnon, Ronald Ian (1973), Money and Capital in Economic Development, Brooking Institutions, Washington. 
Miller, Merton (1998), "Financial Markets and Economic Growth", Journal of Applied Corporate Finance, 11, Univertity of Chicago, pp. 8-14.

Patrick, Hugo (1966), "Financial Development and Economic Growth in Underdeveloped Countries", Economic Development and Cultural Change, 14(2), The University of Chicago Press, Chicago, pp. 174-189.

Ramírez, Rondán y Juan Aquino Chávez (2004), Desarrollo financiero, crisis financiera y acumulación de capital, Pontificia Universidad Católica del Perú, Lima.

Robinson, Joan (1952), The Rate of Interest and other essays, Macmillan, Londres.

Schumpeter, Joseph (1911), The Theory of Economic Development, Librería del Congreso, Washington.

Tenjo, Fernando y Guillermo García (1995), Intermediación financiera y crecimiento económico, Universidad Nacional de Colombia, Bogotá.

Recibido: 24 de junio de 2008. Reenviado: 9 de febrero de 2009.

Aceptado: 6 de mayo de 2009.

Marleny Cardona-Acevedo. Es doctora en ciencias sociales. Es profesorainvestigadora en la Universidad EAfIT de Medellín. Sus líneas actuales de investigación son: estudios sectoriales y territoriales, políticas públicas y Pymes. Entre sus publicaciones destacan: en coautoría, Tecnologías, organización y politicas: mundos de producción de las Pymes en Colombia en el periodo 1990-2002, Universidad de Medellín, Medellín (2007); en coautoría, "Ciudad y violencia: escenarios de homicidios, el caso de Medellín", Revista Cubana Salud Pública, 31(3), La Habana, pp. 203-210 (2005); "Globalización y sindicalismo", Sebastien Arcand et al. (eds.), Sociología de la empresa, del marco histórico a las dinámicas internas, Siglo Hombre Editores-Fondo Editorial Universidad EAFIT-Universidad del Valle, Bogota, pp.163-205 (2010).

Carlos Andrés Cano-Gamboa. Es magíster de economía por la Universidad de Antioquia y economista por la Universidad EAfit (Medellín). 
Actualmente es el coordinador de competitividad y productividad de la Fundación Proantioquia. Es investigador económico del Grupo de Estudios Sectoriales y Territoriales y del Grupo en Economía y Empresa de la Universidad EAfiт. Es profesor de posgrado, pregrado y extensión de la mencionada universidad. Las publicaciones recientes más destacadas son: "Mecanismo de transmisión de las tasas de interés en Colombia (20012007)", Cuadernos de Economía, xxviII (48), Bogotá, pp. 209-241 (2008); "Determinantes en la eficiencia en la producción de educación superior", Revista de la Educación Superior, xxxviI (3), 147, México, pp. 17-31 (2008); "Empleo, desempleo y salario real: análisis del mercado laboral en la ciudad de Medellín (1995-2006)", Lecturas de Economía, 69, (Medellín, pp. 115-139 (2009); Territorio, ciclo de vida y estructura empresarial: un puente en la industrialización regional, Fondo Editorial Universidad EAFIT, Medellín (2005). 